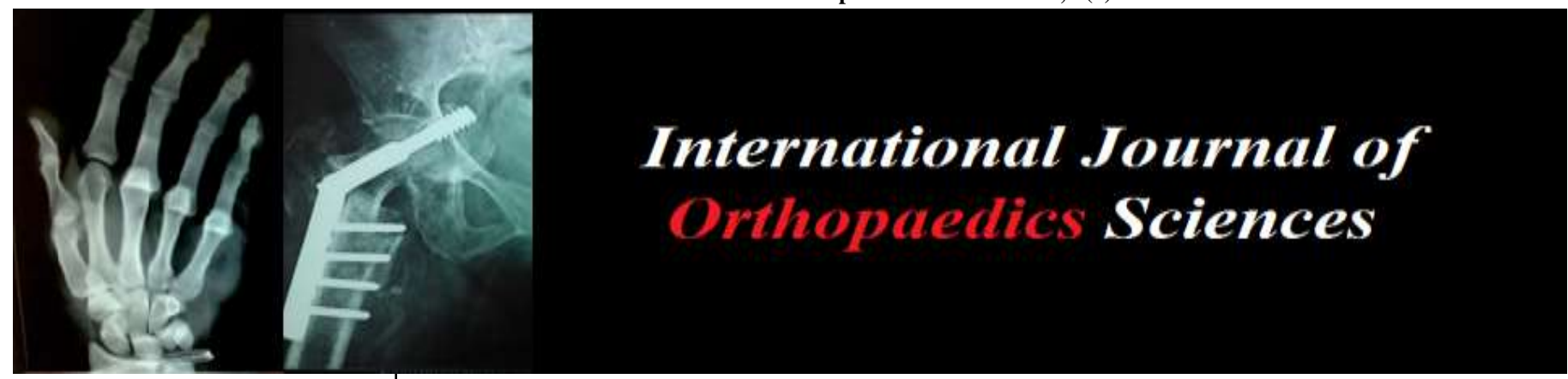

E-ISSN: 2395-1958

P-ISSN: 2706-6630

IJOS 2021; 7(1): 275-277

(C) 2021 IJOS

www.orthopaper.com

Received: 28-10-2020

Accepted: 06-12-2020

Dr. Ashish Kumar Paswan PG Student, Department of Orthopaedics, Darbhanga Medical College and Hospital, Darbhanga, Bihar, India

Dr. Nand Kumar

Professor and H.O.D,

Department of Orthopaedics,

Darbhanga Medical College and

Hospital, Darbhanga, Bihar,

India

Dr. Ashutosh Kumar Assistant Professor,

(Orthopaedics), Department of Trauma and Emergency, Indra Gandi Institute of Medical Sciences, Patna, Bihar, India

Corresponding Author: Dr. Ashutosh Kumar Assistant Professor, (Orthopaedics), Department of Trauma and Emergency, Indira Gandhi Institute of Medical Sciences, Patna, Bihar, India

\section{Comparative study of operative with nonoperative management of calcaneal fracture}

\section{Dr. Ashish Kumar Paswan, Dr. Nand Kumar and Dr. Ashutosh Kumar}

DOI: https://doi.org/10.22271/ortho.2021.v7.i1e.2497

\section{Abstract}

Purpose: The treatment of fractures of the calcaneum remains controversial. There is no consensus in the current literature regarding the optimal treatment of fractures of the calcaneum. In the current study, we intend to compare the outcome of operative and conservative management based on the objective criteria, i.e., restoration of Bohler's angle, subtalar range of motion, and subjective criteria such as pain, return to work, return to physical activity, and change in shoe wear.

Materials and Methods: This is combined retrospective and prospective (from May 2018 to June 2020) study of 25 calcaneum fractures in 24 patients treated in Darbhanga medical college Laheriasarai \& Hospitals. Pretreatment and posttreatment (at follow-up) Bohler's angle were also compared.

Results: Restoration of the Bohler's angle was better with operative management as compared to conservative management. In our study, the results of type I fractures managed conservatively had a better outcome than those of displaced fractures and the difference was statistically significant. Furthermore, type II and type III fractures had a better outcome with operative management, but the difference was not statistically significant. In type IV fractures, operative management was significantly better than conservative management. A significant correlation was seen between the posttreatment Bohler's angle and C-N scores.

Conclusions: Conservative management has better functional outcome for undisplaced fractures. For displaced and comminuted fractures, anatomical reduction and restoration of Bohler's angle is very important. Bohler's angle has a prognostic importance and correlates well with the functional outcome.

Keywords: Bohler's angle, conservative, calcaneum fracture, operative

\section{Introduction}

The calcaneus (os calcis) is the largest and most often fractured tarsal bone. It is the major weight bearing osseous structure of the foot and is one of the components of the tri-tarsal articulation and has important functional tasks with regard to normal ambulation. With a bone so vital to the normal mechanics of locomotion, it is easy to see why a fracture of calcaneus is attended by considerable morbidity. Despite the physician's extensive experience with this injury, its major socio-economic impact in regard to the time lost from work and recreation, and the attention given to it for many years throughout the world, still there is no method of treatment that yields consistently good results. Cave, ${ }^{[1]}$ pointed out that fractures of the calcaneus is one injury that has not increased in frequency with the advent of mechanized industry, automobile travel and war. It has been a common, often disabling injury since humans assumed the erect posture and began to defy gravity. Cotton ${ }^{[2]}$ as early as 1908 wrote, "Ordinarily speaking the man who breaks his heel bone is 'done', so far as his industrial future is concerned. There is great difference of opinion regarding the treatment of these fractures and each method has its ardent proponents and opponents; there is no method applicable for treatment of all these fractures. The methods of treatment that have been proposed are Immobilization in below knee cast. Closed reduction and fixation. Open reduction and internal fixation with or without grafting. Primary subtalar arthrodesis. Immobilization in below knee cast.

Indications for nonoperative treatment include

- Nondisplaced fractures calcaneum

- Fractures in patients with severe peripheral vascular disease or type 1 diabetes 
- Fractures in patients with other medical comorbidities prohibiting surgery

- Elderly patients who are household ambulators

Nonoperative treatment consists of a supportive splint to allow dissipation of the initial fracture hematoma, followed by conversion to a prefabricated fracture boot, with the ankle locked in neutral flexion to prevent an equinous contracture.

Early subtalar and ankle joint range-of-motion exercises are initiated, and non-weight bearing restrictions are maintained for approximately 10 to 12 weeks, until radiographic union is confirmed.

Closed reduction and fixation Simple cast immobilization or early motion and protected weight bearing does not always lead to satisfactory results, particularly in displaced intraarticular fractures of the calcaneus. The aim of closed reduction and fixation is to restore the anatomical configuration of fracture fragments, with emphasis on

Restoring congruity of subtalar joint, Restoring Bohler's angle and restoring the normal width of the calcaneus.

Fixation techniques for tongue-type fractures using a percutaneous nail were first advocated by Westhues. The technique was modified by Gissane, who developed the Gissane spike for this procedure. It was Essex-Lopresti, however, who described the technique in detail and popularized the maneuver specifically for tongue-type fractures. Although different techniques are described the common steps in all the methods are disimpaction of the fracture fragments, reduction of the displaced fragments by manipulation or with percutaneous pins and maintenance of reduction with plaster, pin traction or external fixation.

\section{Essex-Lopresti method}

It was Essex-Lopresti who first distinguished the two most common types of intra- articular calcaneal fractures, the tongue type and the joint depression type.

In tongue type of fractures, he advocated closed reduction with the aid of an axial percutaneous pin. A Steinmann pin is introduced into the tongue fragment in a longitudinal direction angling slightly to the lateral side, under radiological control. The pin should be well into the tongue fragment but not across the fracture site. The idea is to disimpact the fracture by lifting up on one of the pins and simultaneously plantar flexing the foot. Once the fracture is reduced and brought up into position an audible click is heard. At this point the pin is advanced across the fracture into the anterior fragment of the calcaneus. The pin in position is incorporated in a short leg non weight bearing cast for 4-6 weeks. At 6 weeks the pin is removed and the fracture is protected in another short leg cast until clinical and radiological union occurs 10 to 12 weeks. Active range of motion exercises of the ankle and subtalar joint are started after union. Open reduction and bone grafting with or without fixation when operative treatment is selected, it should be performed within the first three weeks after the injury, before early consolidation of the fracture and swelling in the foot and ankle has markedly decreased.

\section{Indications for open reduction and internal fixation with} or without bone grafting

- Young adult or middle aged patients in reasonably good health.

- Displaced posterior and/or middle facet fragments.

- Four or fewer major fragments.

- Sustentaculum tali (anteromedial)

- Posterior facet fragment

- Tuberosity (Posterolateral)

- Lateral wall fragment.
- Fractures with loss of calcaneal height and length.

Which causes the talus to dorsiflex, resulting in impingement of the talus on the anterior tibia.

Loss of length weakens the Achilles tendon plantar flexion mechanism.

\section{Material and Methods}

This study was done to evaluate and compare the results of conservative and operative management for calcaneum fractures on basis of subjective, clinical and radiological criteria in our hospital setup. This is combined retrospective and prospective (from May 2018 to June 2020) study of 25 calcaneum fractures in 24 patients treated in Darbhanga medical college Laheriasarai \& Hospitals. It includes the patients who have already undergone treatment for this fracture and also the patients newly diagnosed as calcaneum fracture. Follow up period ranged from 6 months to 2 years with an average follow up period of 12 months. When the patients were seen for the first time after injury, a thorough history was taken regarding the time of injury, mechanism of injury, any significant past or personal history (especially diabetes and smoking). Patients were examined giving special importance to whether the fracture was open or closed, presence of gross swelling, fracture blisters, features of compartment syndrome and presence of other associated injuries. Routine investigations were done as were necessary. The diagnosis was confirmed by antero-posterior (dorsoplantar), lateral and axial radiographs. CT scan was done to asses the fracture pattern, displacement of fracture fragments, comminution and to classify the fracture according to Sanders classification. Bohler's angle was measured. Open fractures were classified according to Gustilo Anderson classification. Majority of the cases were treated as in-patients and a few as out-patients. The form of treatment, conservative or operative was decided depending on type of fracture, patients age, condition of soft tissues, associated injuries, comorbidities, patients occupation, affordability as well as surgeon's decision. Out of fractures, were managed conservatively and 12 were surgically managed. Conservative management was in a form of below knee plaster cast initially if there was no gross swelling. If there was a swelling present, first it was reduced by limb elevation, local ice packs and anti-inflammatory drugs and once the swelling reduced then cast was put. All the patients were advised NWB walking. Cast was removed after 6-8 weeks of application. Patients were assessed for fracture union radiologically. Once the fracture has united, partial weight bearing was advised which was gradually increased to full weight bearing. The main indication for operative management was displaced intraarticular fractures with incongruous subtalar joint and reduced Bohler's angle. The method of reduction and fixation were decided according to the fracture pattern, the surgeons experience, associated injuries and affordability of the patient. Closed reduction with or without fixation was done within 2448 hours of injury and open reduction and fixation was done once the skin condition was good but within 3 weeks of injury. The basic idea was to achieve near anatomical reduction and a congruous subtalar joint. Essex-lopresti reduction was done in 2 cases. With patients in prone position under $\mathrm{C}$ arm guidance a Steinmann pin was inserted through the posterior end of calcaneus into the tongue fragment. This fragment was levered into reduced position using a pin. Then pin was driven into anterior process. A below knee cast was applied following this. Closed reduction and $\mathrm{K}$ wire fixation was done for 3 patients. In this after achieving the reduction and maintaining the Bohler's angle, the reduction was secured 
with $\mathrm{K}$ wires. $\mathrm{B} \mathrm{K}$ cast was put following this. In both the above methods, at end of 6 weeks cast and pins were removed and check x-rays were taken to assess fracture union. Open reduction and internal fixation was used in 3 patients. It was done as a planned and elective procedure.

Before patients were taken up for surgery they were put on foot elevation, local ice packs, glycerine magsulf dressing and anti-inflammatory drugs for few days to reduce foot swelling. For all 3 cases Seligson's lateral approach was used. The fracture was reduced and temporarily fixed with $\mathrm{K}$ wires, if needed. The definitive fixation was done with help of calcaneum plate in 2 cases and reconstruction plate in 1 case. Post operatively limbs were immobilized in plaster cast, patients were put on antibiotics and analgesics and limbs elevated. Wounds were inspected on third post operative day by creating a window over the wound in the cast and sutures were removed on average on twelfth day (decided according to the wound inspection). Once the wound were healed well, window was closed and cast reinforced. Patients were advised strict non-weight with axillary crutches and then discharged. Cast was removed at 6 weeks and fracture was assessed radiologically for union. Physiotherapy exercises for of ankle and sub-talar movements were started on removal of cast. Once the fracture united radiologically, patients were started on partial weight bearing walking which was gradually increased to full weight bearing. There were 7 open calcaneum fractures, 2 of which were managed conservatively as the fracture fragments were undisplaced (Sander's type I fracture). For one patient with open IIIA injury with ankle subluxation after wound debridement, fracture and ankle was stabilized using A-O external fixator and wound was covered with SSG. For another patient with open IIIA injury with medial malleolus fracture JESS was applied and wound covered with SSG. For one patient with open II injury with undisplaced talus and navicular fracture A-O external fixator was applied. There were two open I injuries out of which CRIF with $\mathrm{K}$ wires was done and for another ORIF with reconstruction plate was done. For all the patients with open fractures after thorough debridement and washing with saline, povidone iodine and Hydrogen peroxide, adequate I.V antibiotics cover was given. For 5 out of 7 patients primary closure was done and for another 2 patients with open IIIA injury SSG was applied as a second sitting procedure after 48 hours.

\section{Results}

A total of 25 cases were enrolled in the study .Majority of the patients were in age group of 30-50 years. Mean age in both the groups was similar.

All type I ie undisplaced fractures were treated conservatively. In type II were treated equally. Majority of type III and type IV were treated surgically.

In our series, with conservative management most of the patients had good outcome with type I fractures, fair outcome with type II fractures, poor outcome with type III and type IV fractures. In the operative group, among the patients with type II fractures, one patient had good result and other had excellent result. With type III fracture most of them had fair result and with type IV fracture most of them had good results which were statistically significant ( $p$ value- 0.03 ).

\section{Discussion}

The treatment of calcaneal fractures remains controversial. In this study the outcomes of operatively managed and conservatively managed calcaneal fractures was compared in order to find out the better method for different type of fracture. Two different parameters were used to compare the outcome and finally correlation between the two parameters was evaluated. The results of present study are compared with those of previous similar studies.

\section{Summary}

A combined retrospective and prospective study was conducted on patients with calcaneum fractures .A total of 24 patients with fractures were included in the study, out of which 13 fractures were managed conservatively and 12 were managed surgically.

Outcome was evaluated on basis of functional outcome and radiological assessment. Functional outcome was assessed on basis of Creighton-Nebraska health foundation scoring system and radiological assessment was done by comparing pre and post treatment (at follow up of minimum 6 months) Bohler's angle. In our study, undisplaced fractures managed conservatively had a good functional outcome. Displaced and comminuted fractures managed surgically had a relatively better outcome as compared to conservative management provided Bohler's angle was restored. In view of the fact that the numbers of cases were limited in this study, it would be difficult to come to any definite conclusion. But our study clearly suggests that restoration of Bohler's angle is the key to the final outcome, irrespective of the method of treatment. We feel that our results of surgery are letter since it is difficult to reduce the fracture and restore the Bohler's angle in a conservative managed cases as compared to open reduction in displaced types of fracture. Hence we conclude that Bohler's angle has a prognostic importance and correlates with the functional outcome. According to Paul et al. and D Makki et al. restoration of Bohler's angle was associated with better outcome. Our study also reemphasizes that prompt osteosynthesis should be considered for intra-articular fractures of calcaneum in order to restore the shape of hindfoot and Bohler's angle. A study with a larger sample size, with more number of cases in each group is required to make definite recommendations.

\section{References}

1. Cave JB. Mechanism and patho anatomy of intraarticular calcaneal fracture. Clin Orthery 1993;290:3-6.

2. Cotton FJ. Fractures of the os calcis. Boston Med Surg J 1908; 18:559-565.

3. Bankart, Massart P, Delvaux D. The operative treatment of intraarticular calcaneal fractures: indications, technique and results in 257 cases. Clin Orthop 1993;290:55-58.

4. Conn HR. The treatment of fractures of the os calcis. J Bone Joint Surg 1935;17:392-405.

5. Bellabarba C, sangeorzan BJ. Fractures of the calcaneus. Orthop Clin North Am 1999;33:263-266.

6. Koval K, Sanders R. The radiologic evaluation of calcaneal fractures. Clin Orthop 1993;290:41-58.

7. Cave JB. Mechanism and path anatomy of intra- articular calcaneal fracture. Clin Orthery 1993;290:3-6.

8. Miric A, Patterson BM. Path anatomy of intraarticular fractures of the calcaneum. J Bone Joint Surg 1998;80A:207-218.

9. Essex-Lopresti P. The mechanism, reduction technique and results in fractures of the os calcis. $\mathrm{Br} \mathrm{J}$ surg 1952;39:395-415.

10. Displaced Intraarticular calcaneal fractures: results using A prognostic computed tomography scan classification Clin Orthop 1993. 